FERMILAB-Pub-01/228-T

hep-ph/0108070

October 2001

\title{
GUT Model Predictions for Neutrino Oscillation Parameters Compatible with the Large Mixing Angle Solar Solution
}

\author{
Carl H. Albright ${ }^{1,2}$ and S. Geer ${ }^{2}$ \\ ${ }^{1}$ Department of Physics, Northern Illinois University, DeKalb, IL 60115 \\ ${ }^{2}$ Fermi National Accelerator Laboratory, P.O. Box 500, Batavia, IL 60510, USA
}

\begin{abstract}
Within the framework of an $\mathrm{SO}(10)$ GUT model that can accommodate both the atmospheric and the LMA solar neutrino mixing solutions, we present explicit predictions for the neutrino oscillation parameters $\sin ^{2} 2 \theta_{13}$, $\sin ^{2} 2 \theta_{12}, \sin ^{2} 2 \theta_{23}$, and $\Delta m_{21}^{2}$. Precise measurements of $\sin ^{2} 2 \theta_{12}$ and $\Delta m_{21}^{2}$ by KamLAND can be used to precisely determine the GUT model parameters. We find that the model can then be tested at Neutrino Superbeams and Neutrino Factories with precision neutrino oscillation measurements of $\sin ^{2} 2 \theta_{23}, \sin ^{2} 2 \theta_{13}$, and the leptonic CP phase $\delta_{C P}$.
\end{abstract}

PACS numbers: 12.15.Ff, 12.10.Dm, 12.60.Jv, 14.60.Pq

Electronic addresses: albright@fnal.gov, sgeer@fnal.gov 


\section{INTRODUCTION}

Over the last few years the evidence for neutrino oscillations between the three known active-neutrino flavors $\left(\nu_{e}, \nu_{\mu}\right.$, and $\left.\nu_{\tau}\right)$ has become increasingly convincing. The atmospheric neutrino flux measurements from the Super-Kamiokande (Super-K) experiment exhibit a deficit of muon neutrinos which varies with zenith angle (and hence baseline) in a way consistent with $\nu_{\mu} \rightarrow \nu_{x}$ oscillations [1]. In principle $\nu_{x}$ could be $\nu_{e}, \nu_{\tau}, \nu_{s}$ (where $\nu_{s}$ is a light sterile neutrino), or some combination of these. However, further Super-K measurements exclude $\nu_{x}$ being predominantly $\nu_{s}$, and reactor $\nu_{e}$ disappearance results from the CHOOZ experiment [2] exclude $\nu_{x}$ being predominantly $\nu_{e}$. Hence, the Super-K atmospheric neutrino measurements provide strong evidence for $\nu_{\mu} \rightarrow \nu_{\tau}$ oscillations; indeed there is some evidence for $\nu_{\tau}$ interactions in the Super-K data. In addition to the atmospheric neutrino deficit, there has been the long-standing result, first obtained from the Homestake experiment [3], that the $\nu_{e}$ flux from the sun is less than expected. The recent measurement of the total flux of active neutrinos from the sun obtained from the SNO experiment [四] is consistent with the predicted flux from solar models [5]. Hence, when taken together with solar neutrino measurements from Super-K [6], the SNO results imply that there is a component of active neutrinos within the solar flux that is not $\nu_{e}$, and hence that $\nu_{e} \rightarrow \nu_{x}$ oscillations are taking place, where $\nu_{x}$ can be $\nu_{\mu}$ and/or $\nu_{\tau}$. The solar neutrino and atmospheric neutrino results, taken together, suggest that oscillations occur between all three known active flavors.

The atmospheric neutrino data are consistent with $\nu_{\mu} \rightarrow \nu_{\tau}$ oscillations provided the oscillation parameters that define the oscillation amplitude and frequency lie in one welldefined region of parameter space. In contrast, the solar neutrino measurements are currently consistent with the associated oscillation parameters being within any of four regions of parameter space. However, although the evidence is not yet compelling, the data seem to exhibit a preference for one of these regions of parameter space, namely the one corresponding to the Large Mixing Angle (LMA) MSW solution [7].

The splittings between the squares of the masses of the neutrino mass eigenstates determine the oscillation frequency. The atmospheric- and solar-neutrino oscillation data imply that neutrinos have masses in the range $10^{-5}-1 \mathrm{eV}$. This mass scale can be accommodated naturally within the framework of models based on Grand Unified Theories (GUTs). The very small neutrino mass is easily generated by the seesaw mechanism [8] in which the light neutrino mass matrix is obtained from the Dirac and right-handed Majorana neutrino mass matrices.

Grand Unified models provide a theory of flavor, and relate quark masses and mixings to lepton masses and mixings. Hence, neutrino oscillation data, which measure neutrino masses and mixings, constrain GUT models. In this paper, for one promising GUT model, we explore how future neutrino oscillation experiments can test the theory. We restrict ourselves to the LMA solution for the solar neutrino data, and provide predictions for the neutrino mass-splittings and mixing angles that will be measured in the next few years. 


\section{THREE-FLAVOR MIXING}

Within the framework of three-flavor mixing, the flavor eigenstates $\nu_{\alpha}(\alpha=e, \mu, \tau)$ are related to the mass eigenstates $\nu_{j}(j=1,2,3)$ in vacuum by

$$
\nu_{\alpha}=\sum_{j} U_{\alpha j} \nu_{j}
$$

where $U$ is the unitary $3 \times 3$ Maki-Nakagawa-Sakata (MNS) mixing matrix 9] times a diagonal phase matrix $\Phi_{M}: U=U_{M N S} \Phi_{M}$. The MNS mixing matrix is conventionally specified by 3 mixing angles $\left(\theta_{23}, \theta_{12}, \theta_{13}\right)$ and a $\mathrm{CP}$-violating phase $\left(\delta_{C P}\right)$ with the parameterization

$$
U_{M N S}=\left(\begin{array}{ccc}
c_{12} c_{13} & s_{12} c_{13} & s_{13} e^{-i \delta_{C P}} \\
-s_{12} c_{23}-c_{12} s_{23} s_{13} e^{i \delta_{C P}} & c_{12} c_{23}-s_{12} s_{23} s_{13} e^{i \delta_{C P}} & s_{23} c_{13} \\
s_{12} s_{23}-c_{12} c_{23} s_{13} e^{i \delta_{C P}} & -c_{12} s_{23}-s_{12} c_{23} s_{13} e^{i \delta_{C P}} & c_{23} c_{13}
\end{array}\right),
$$

where $c_{j k} \equiv \cos \theta_{j k}$ and $s_{j k} \equiv \sin \theta_{j k}$. The angles can be restricted to the first quadrant, $0 \leq \theta_{i j} \leq \pi / 2$, with $\delta_{C P}$ in the range $-\pi \leq \delta_{C P} \leq \pi$, though it will later prove advantageous to consider $\theta_{13}$ in the fourth quadrant. The $\Phi_{M}$ phase matrix has the form

$$
\Phi_{M}=\operatorname{diag}\left(e^{i \chi_{1}}, e^{i \chi_{2}}, 1\right),
$$

where $\chi_{1}$ and $\chi_{2}$ are Majorana phases which can not be rotated away.

The atmospheric neutrino oscillation data indicate that [1]

$$
\begin{aligned}
\left|\Delta m_{32}^{2}\right| & \simeq 3.2 \times 10^{-3} \mathrm{eV}^{2}, \\
\sin ^{2} 2 \theta_{23} & =1.0,(\geq 0.89 \text { at } 90 \% \text { c.l. }),
\end{aligned}
$$

where $\Delta m_{i j}^{2} \equiv m_{i}^{2}-m_{j}^{2}$ and $m_{1}, m_{2}$ and $m_{3}$ are the mass eigenstates.. The atmospheric neutrino oscillation amplitude can be expressed in terms of the $U_{M N S}$ matrix elements and is given by $\sin ^{2} 2 \theta_{a t m}=4\left|U_{\mu 3}\right|^{2}\left(1-\left|U_{\mu 3}\right|^{2}\right) \simeq 4\left|U_{\mu 3}\right|^{2}\left|U_{\tau 3}\right|^{2}$. The approximation is valid because $\left|U_{e 3}\right|$ is known to be small [2].

The solar neutrino oscillation data from Super-K indicate that, for the LMA solution, the allowed region is approximately bounded by

$$
\begin{aligned}
\Delta m_{21}^{2} & =(2.2-17) \times 10^{-5} \mathrm{eV}^{2}, \\
\sin ^{2} 2 \theta_{\text {sol }} & =(0.6-0.9),
\end{aligned}
$$

where the solar neutrino oscillation amplitude is given by $\sin ^{2} 2 \theta_{\text {sol }}=4\left|U_{e 1}\right|^{2}\left(1-\left|U_{e 1}\right|^{2}\right) \simeq$ $4\left|U_{e 1}\right|^{2}\left|U_{e 2}\right|^{2}$. In defining the viable region of GUT model parameter space we shall make use of the allowed LMA solar mixing region specified in [6]. Other recent analyses also prefer the LMA solution [10]. 


\section{THE GUT MODEL}

The GUT model which shall be studied here was developed by Albright and Barr 11 and is based on the grand unified group $S O(10)$ with a $U(1) \times Z_{2} \times Z_{2}$ flavor symmetry. We adopt this model in our present study because it can accommodate the LMA solution and makes quantitative predictions for the measured oscillation parameters. The model involves a minimum set of Higgs fields which solves the doublet-triplet splitting problem. This requires just one $\mathbf{4 5} \mathbf{5}_{H}$ whose $\mathrm{VEV}$ points in the $B-L$ direction, and there are no higher rank representations. Two pairs of $\mathbf{1 6}_{H}, \overline{\mathbf{1 6}}_{H}$ 's stabilize the solution [12]. Several Higgs in the $\mathbf{1 0}_{H}$ representations, together with Higgs singlets, are also present. The Higgs superpotential exhibits the $U(1) \times Z_{2} \times Z_{2}$ symmetry [12 which is used for the flavor symmetry of the GUT model. The combination of VEVs, $\left\langle\mathbf{4} \mathbf{5}_{\mathbf{H}}\right\rangle_{B-L},\left\langle 1\left(\mathbf{1 6}_{\mathbf{H}}\right)\right\rangle$ and $\left\langle 1\left(\overline{\mathbf{1 6}}_{\mathbf{H}}\right)\right\rangle$ break $S O(10)$ to the Standard Model. The electroweak VEVs arise from the combinations $v_{u}=\left\langle 5\left(\mathbf{1 0}_{\mathbf{H}}\right)\right\rangle$ and $v_{d}=\left\langle\overline{5}\left(\mathbf{1 0}_{\mathbf{H}}\right)\right\rangle \cos \gamma+\left\langle\overline{5}\left(\mathbf{1 6}_{\mathbf{H}}^{\prime}\right)\right\rangle \sin \gamma$, while the combination orthogonal to $v_{d}$ gets massive at the GUT scale. As such, Yukawa coupling unification can be achieved at the GUT scale with $\tan \beta \sim 2-55$, depending upon the $\overline{5}\left(\mathbf{1 0}_{\mathbf{H}}\right)-\overline{5}\left(\mathbf{1 6}_{\mathbf{H}}\right)$ mixing present for the $v_{d} \mathrm{VEV}$. In addition, matter superfields appear in the following representations: $16_{1}, \mathbf{1 6}_{\mathbf{2}}, \mathbf{1 6 _ { 3 }} ; \mathbf{1 6}, \overline{\mathbf{1 6}}, \mathbf{1 6} \mathbf{6}^{\prime}, \overline{\mathbf{1 6}}, \mathbf{1 0}_{\mathbf{1}}, \mathbf{1 0}_{\mathbf{2}}$, and $\mathbf{1}$ 's, where all but the $\mathbf{1 6}_{\mathbf{i}}(i=1,2,3)$ get superheavy and are integrated out.

The Dirac mass matrices for the up quarks, down quarks, neutrinos and charged leptons are found to be

$$
\begin{aligned}
& U=\left(\begin{array}{ccc}
\eta & 0 & 0 \\
0 & 0 & \epsilon / 3 \\
0 & -\epsilon / 3 & 1
\end{array}\right) M_{U}, \quad D=\left(\begin{array}{ccc}
0 & \delta & \delta^{\prime} e^{i \phi} \\
\delta & 0 & \sigma+\epsilon / 3 \\
\delta^{\prime} e^{i \phi} & -\epsilon / 3 & 1
\end{array}\right) M_{D}, \\
& N=\left(\begin{array}{ccc}
\eta & 0 & 0 \\
0 & 0 & -\epsilon \\
0 & \epsilon & 1
\end{array}\right) M_{U}, \quad L=\left(\begin{array}{ccc}
0 & \delta & \delta^{\prime} e^{i \phi} \\
\delta & 0 & -\epsilon \\
\delta^{\prime} e^{i \phi} & \sigma+\epsilon & 1
\end{array}\right) M_{D}
\end{aligned}
$$

where

$$
\begin{aligned}
M_{U} & \simeq 113 \mathrm{GeV}, & M_{D} & \simeq 1 \mathrm{GeV}, \\
\sigma & =1.78, & \epsilon & =0.145, \\
\delta & =0.0086, & \delta^{\prime} & =0.0079, \\
\phi & =126^{\circ}, & \eta & =8 \times 10^{-6}
\end{aligned}
$$

are input parameters defined at the GUT scale to fit the low scale observables after evolution downward from $\Lambda_{G U T}$. Note that the phase $\phi$ was incorrectly stated as $54^{\circ}$ in [11]. The above textures were obtained by imposing the Georgi-Jarlskog relations [13] at $\Lambda_{G U T}$, $m_{s}^{0} \simeq m_{\mu}^{0} / 3, m_{d}^{0} \simeq 3 m_{e}^{0}$ with Yukawa coupling unification holding for $\tan \beta \sim 5$. The matrix element contributions can be understood in terms of Froggatt-Nielsen diagrams [14 as explained in [1].

All nine quark and charged lepton masses, plus the three CKM angles and CP phase, are well-fitted with the eight input parameters. With no extra phases present, aside from the one appearing in the CKM mixing matrix, the vertex of the CKM unitary triangle occurs at the center of the presently allowed region with $\sin 2 \beta \simeq 0.64$. The Hermitian 
matrices $U^{\dagger} U, D^{\dagger} D$, and $N^{\dagger} N$ are diagonalized with small left-handed rotations, while $L^{\dagger} L$ is diagonalized by a large left-handed rotation. This accounts for the small value of $V_{c b}=\left(U_{U}^{\dagger} U_{D}\right)_{c b}$, while $\left|U_{\mu 3}\right|=\left|\left(U_{L}^{\dagger} U_{\nu}\right)_{\mu 3}\right|$ will turn out to be large for any reasonable righthanded Majorana mass matrix, $M_{R}$ [15].

The effective light neutrino mass matrix, $M_{\nu}$, is obtained from the seesaw mechanism [8] whereby $M_{\nu}=N^{T} M_{R}^{-1} N$. While the large atmospheric neutrino mixing $\nu_{\mu} \leftrightarrow \nu_{\tau}$ arises primarily from the structure of the charged lepton mass matrix, the solar and atmospheric mixings are essentially decoupled in the model, so the structure of the right-handed Majorana mass matrix determines the type of $\nu_{e} \leftrightarrow \nu_{\mu}, \nu_{\tau}$ solar neutrino mixing. Any one of the recently favored four solar neutrino mixing solutions can be obtained. The LMA solution relevant to our study here requires some fine-tuning and a hierarchical structure, but this can be explained in terms of Froggatt-Nielsen diagrams. The most general form for the right-handed Majorana mass matrix we consider is [11]

$$
M_{R}=\left(\begin{array}{ccc}
c^{2} \eta^{2} & -b \epsilon \eta & a \eta \\
-b \epsilon \eta & \epsilon^{2} & -\epsilon \\
a \eta & -\epsilon & 1
\end{array}\right) \Lambda_{R}
$$

where the parameters $\epsilon$ and $\eta$ are those introduced in Eq.(6) for the Dirac sector. Note that the 2-3 subsector has zero determinant and is closely related to that of $N$, as can also be understood in terms of Froggatt-Nielsen diagrams. If we set $a=b=c$, there is just one hierachy present involving one Higgs singlet which induces a $\Delta L=2$ transition. In this case the determinant of $M_{R}$ vanishes. In order to have an invertible $M_{R}$ and a viable seesaw mechanism, for simplicity we set $b=c$ but choose $a \neq b$. This is neatly explained in terms of two Higgs singlets which break lepton number. One singlet contributes to all nine matrix elements while, by virtue of its flavor charge assignment, the other singlet modifies only the 13 and 31 elements of $M_{R}$.

To obtain $U_{M N S}$ from the mass matrices $L$ and $M_{\nu}$, we compute the unitary transformations $U_{L}$ and $U_{\nu}$ that diagonalize $L^{\dagger} L$ and $M_{\nu}^{\dagger} M_{\nu}$ and yield the squares of the charged and neutral lepton mass eigenvalues, respectively. Three arbitrary phase transformations can be performed on the columns of $U_{L}$ which are constructed from the eigenvectors of $L^{\dagger} L$. However, since $M_{\nu}$ is complex symmetric, it can also be diagonalized by use of the same $U_{\nu}$ :

$$
U_{\nu}^{T} M_{\nu} U_{\nu}=\operatorname{diag}\left(m_{1},-m_{2}, m_{3}\right) .
$$

Since we want the light neutrino masses to be real, $U_{\nu}$ can not be arbitrarily phase transformed and is uniquely specified up to sign changes on its column eigenvectors. The unitary mixing matrix $U$ in Eq. (1) is then given by

$$
U=U_{M N S} \Phi_{M}=\left(\Phi_{\text {row }}^{\dagger} U_{L}^{\dagger} U_{\nu} \Phi_{c o l}\right) \Phi_{c o l}^{\dagger},
$$

where $\Phi_{\text {row }}$ and $\Phi_{\text {column }}$ are the row and column phase transformations

$$
\begin{aligned}
\Phi_{\text {row }} & =\operatorname{diag}\left(e^{-i \phi_{1}}, e^{-i \phi_{2}}, e^{-i \phi_{3}}\right), \\
\Phi_{\text {col }} & =\operatorname{diag}\left(e^{-i \chi_{1}}, e^{-i \chi_{2}}, 1\right)
\end{aligned}
$$


of $U_{L}^{\dagger} U_{\nu}$ needed to bring $U_{M N S}$ into the parametric form of Eq.(2) whereby the $e 1, e 2, \mu 3$ and $\tau 3$ elements are real and positive, the real parts of $\mu 2$ and $\tau 1$ are positive, while the real parts of $\mu 1$ and $\tau 2$ are negative. The last factor $\Phi_{c o l}^{\dagger}$ serves to undo the column phase transformation on $U_{\nu}$ and is just the Majorana phase matrix, $\Phi_{M}=\Phi_{c o l}^{\dagger}$, from which the two Majorana phases $\chi_{1}$ and $\chi_{2}$ can be extracted. As noted above, one is free to replace individually the column vectors of $M_{\nu}$ by their negatives, so the Majorana phases have a $180^{\circ}$ ambiguity. Finally, the leptonic CP phase $\delta_{C P}$ can be identified from the $e 3$ element of $U_{M N S}$ or alternatively by constructing the Jarlskog invariant [16], $J=\operatorname{Im}\left(U_{e 2} U_{e 3}^{*} U_{\mu 2}^{*} U_{\mu 3}\right)$, of the untransformed $U_{L}^{\dagger} U_{\nu}$ matrix. The quadrant in which the phase $\delta_{C P}$ lies is uniquely determined once the $\operatorname{sign}$ of $\sin \theta_{13}$ is specified. In carrying out the phase transformations, we have reduced the six inherent phase factors in $U_{L}^{\dagger} U_{\nu}$ to just three physical ones, $\phi_{C P}, \chi_{1}$ and $\chi_{2}$.

As an example, with $a=1, b=c=2$ and $\Lambda_{R}=2.4 \times 10^{14} \mathrm{GeV}$, the seesaw mechanism results in the light neutrino mass matrix

$$
M_{\nu}=N^{T} M_{R}^{-1} N=\left(\begin{array}{ccc}
0 & -\epsilon & 0 \\
-\epsilon & 0 & 2 \epsilon \\
0 & 2 \epsilon & 1
\end{array}\right) M_{U}^{2} / \Lambda_{R}
$$

with three texture zeros. We obtain

$$
\begin{array}{ll}
m_{1}=5.6 \times 10^{-3}, \quad m_{2}=9.8 \times 10^{-3}, \quad m_{3}=57 \times 10^{-3} \mathrm{eV}, \\
M_{1}=M_{2}=2.8 \times 10^{8} \mathrm{GeV}, & M_{3}=2.5 \times 10^{14} \mathrm{GeV}, \\
\Delta m_{32}^{2}=3.2 \times 10^{-3} \mathrm{eV}^{2}, & \sin ^{2} 2 \theta_{\text {atm }}=0.994, \\
\Delta m_{21}^{2}=6.5 \times 10^{-5} \mathrm{eV}^{2}, & \sin ^{2} 2 \theta_{\text {sol }}=0.88, \\
U_{e 3}=-0.01395-0.00085 i, & \sin ^{2} 2 \theta_{\text {reac }}=0.0008 . \\
J=2.0 \times 10^{-4}, \quad \delta_{C P}=-3.5^{\circ}, & \chi_{1}=-0.2^{\circ}, \quad \chi_{2}=0.1^{\circ} .
\end{array}
$$

Here we have chosen the convention $\sin \theta_{13}<0$, so that the $\mathrm{CP}$ phase $\delta_{C P}$ is near zero rather than $180^{\circ}$. The small value of $\delta_{C P}$ follows since $M_{\nu}$ is real in this example, while $L$ contributes only a small phase contribution. The two Majorana phases are very small, since essentially no phase rotation on the right is needed to bring $U_{L}^{\dagger} U_{\nu}$ into the standard MNS form of Eq. (2). The effective neutrinoless double beta decay mass is given by

$$
\left\langle m_{\beta \beta}\right\rangle=\left|\sum_{i} m_{i} U_{e i}^{2}\right|=5.7 \times 10^{-4} \mathrm{eV}
$$

where the Majorana phases and the signs of the eigenvalues in Eq. (9) are taken into account. In the GUT model we are considering, $\left\langle m_{\beta \beta}\right\rangle \sim$ few $\times 10^{-4} \mathrm{eV}$ is obtained over the entire viable LMA region. Note that these values will not be accessible to the presently planned double beta decay experiments.

The above results compare favorably with the determination of the atmospheric neutrino mixing parameters by the Super-K collaboration as given in Eq. (4), as well as their present best-fit point in the solar neutrino LMA region as given in Eq. (5). In fact, the whole presently-allowed LMA region [10] can be covered with $1.0 \lesssim a \lesssim 2.4$ and $1.8 \lesssim b=c \lesssim 5.2$. 


\section{RESULTS}

We can now examine the viable region of GUT model parameter space that is consistent with the LMA solar neutrino solution and explore the predicted relationships between the observables $\sin ^{2} 2 \theta_{23}, \sin ^{2} 2 \theta_{12}, \sin ^{2} 2 \theta_{13}, \delta_{C P}, \Delta m_{32}^{2}$, and $\Delta m_{21}^{2}$. We will first consider the simplest case in which there are, in effect, only two real dimensionless GUT model parameters. We then look at the more general case in which we allow a finite phase $\phi^{\prime}$ so that $a$ is complex.

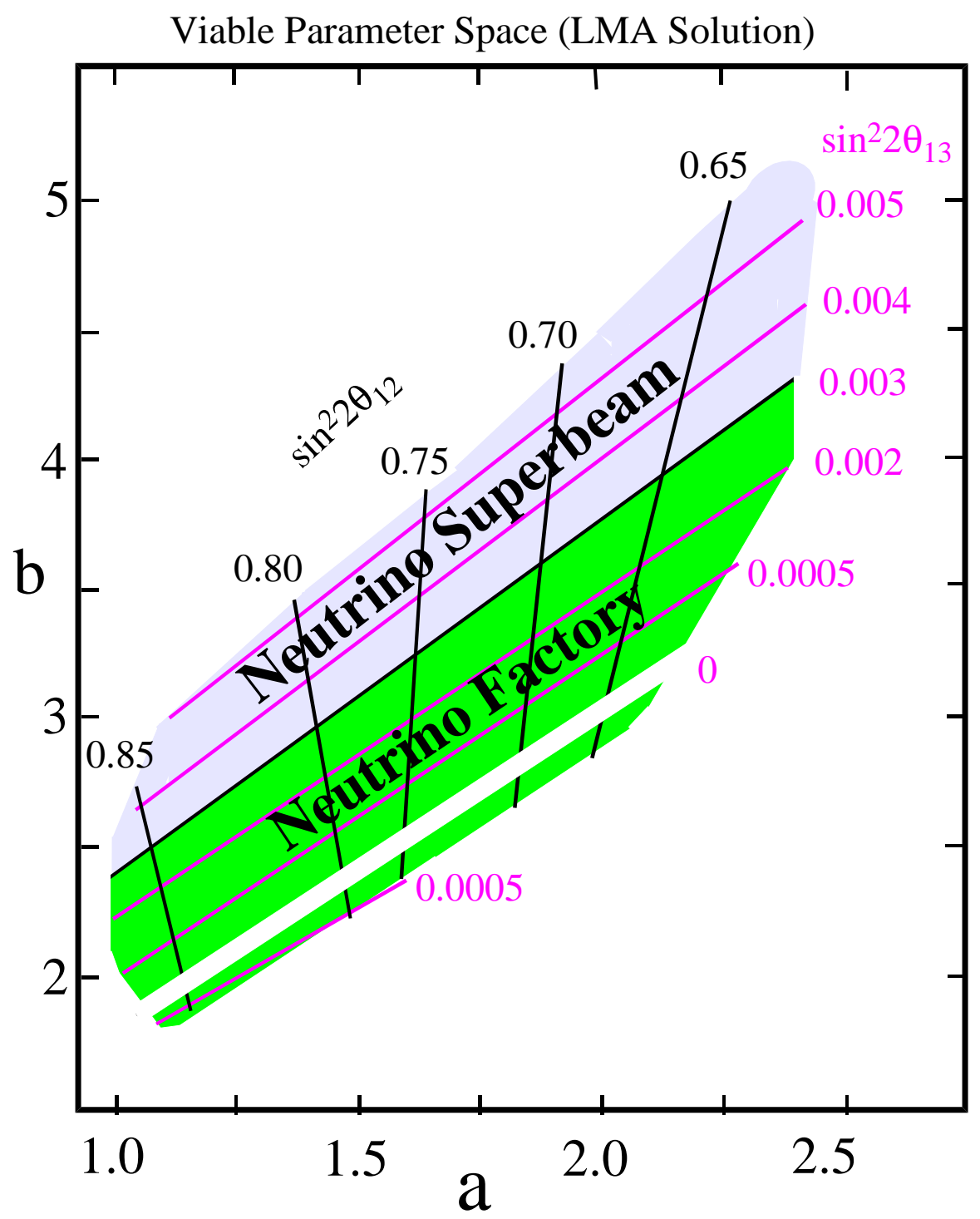

FIG. 1. The viable region of GUT parameter space consistent with the present bounds on the LMA MSW solution. Contours of constant $\sin ^{2} 2 \theta_{13}$ and lines of constant $\sin ^{2} 2 \theta_{12}$ are shown. The region above $\sin ^{2} 2 \theta_{13}=0.003$ can be explored with Neutrino Superbeams, while the region below this can be explored with Neutrino Factories, down to $\sin ^{2} 2 \theta_{13} \sim 0.0001$. 


\section{A. Parameter Choice: $a$ and $b=c$ Real}

The viable region of GUT model parameter space consistent with the LMA solar solution is shown in Fig. 1. Both parameters $a$ and $b$ are constrained by the data to be close to unity, with $1.0 \lesssim a \lesssim 2.4$ and $1.8 \lesssim b \lesssim 5.2$. Superimposed on the allowed region, Fig. 1 1 shows contours of constant $\sin ^{2} 2 \theta_{12}$ (which are approximately parallel to the $b$-axis) and contours of constant $\sin ^{2} 2 \theta_{13}$ (which are approximately at $45^{\circ}$ in the $(a, b)$-plane).

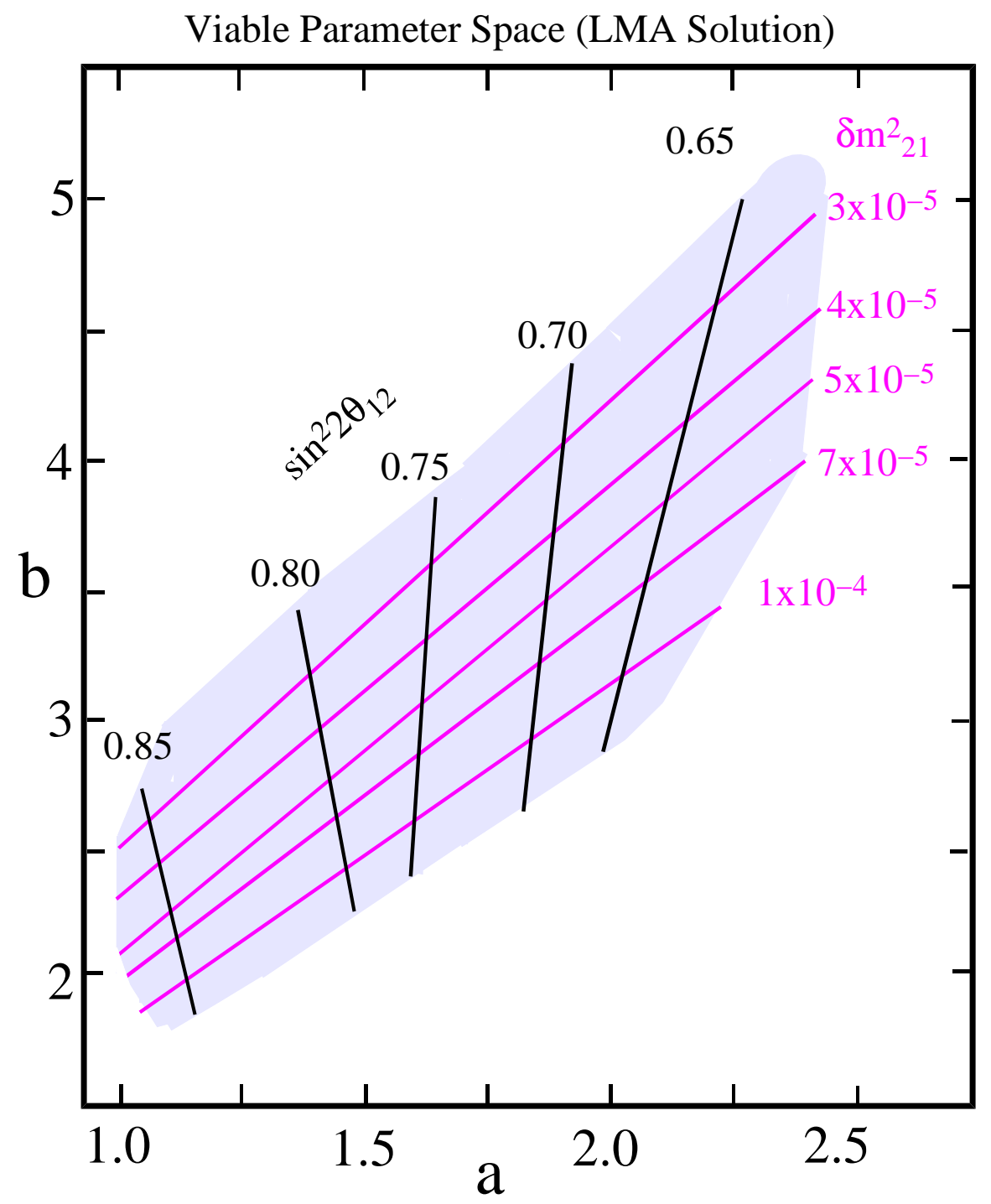

FIG. 2. The viable region of GUT parameter space consistent with the present bounds on the LMA MSW solution. Contours of constant $\Delta m_{21}^{2}$ and lines of constant $\sin ^{2} 2 \theta_{12}$ are shown.

The coming long-baseline accelerator neutrino oscillation experiment MINOS [17] at Fermilab, and the CNGS experiments [18] at CERN, are expected to be able to observe a $\nu_{\mu} \rightarrow \nu_{e}$ signal if $\sin ^{2} 2 \theta_{13}>0.03$. This is above the allowed region of the $(a, b)$-parameter space. Hence the GUT model we are considering predicts that these long-baseline experi- 
TABLE I. List of eight points selected in the LMA allowed parameter region to illustrate the neutrino oscillation parameter predictions of the GUT model.

\begin{tabular}{|c|c|c|c|c|c|c|c|}
\hline \multirow[t]{2}{*}{ Point } & \multicolumn{2}{|c|}{ Model Parameters } & \multirow{2}{*}{$\begin{array}{c}\Delta m_{21}^{2} \\
\mathrm{eV}^{2}\end{array}$} & \multirow{2}{*}{$\begin{array}{c}\Delta m_{32}^{2} \\
\mathrm{eV}^{2}\end{array}$} & \multirow[t]{2}{*}{$\sin ^{2} 2 \theta_{12}$} & \multirow[t]{2}{*}{$\sin ^{2} 2 \theta_{23}$} & \multirow[t]{2}{*}{$\sin ^{2} 2 \theta_{13}$} \\
\hline & $a$ & $b$ & & & & & \\
\hline$(\mathrm{A})$ & 1.0 & 2.0 & $6.5 \times 10^{-5}$ & $3.2 \times 10^{-3}$ & 0.880 & 0.994 & 0.0008 \\
\hline (B) & 1.2 & 2.8 & $3.2 \times 10^{-5}$ & $3.2 \times 10^{-3}$ & 0.838 & 0.980 & 0.0038 \\
\hline (C) & 1.7 & 2.7 & $10.9 \times 10^{-5}$ & $3.2 \times 10^{-3}$ & 0.732 & 0.996 & 0.00008 \\
\hline (D) & 1.7 & 3.0 & $6.3 \times 10^{-5}$ & $3.2 \times 10^{-3}$ & 0.745 & 0.999 & 0.0014 \\
\hline (E) & 1.7 & 3.4 & $4.0 \times 10^{-5}$ & $3.2 \times 10^{-3}$ & 0.747 & 0.992 & 0.0033 \\
\hline$(\mathrm{F})$ & 2.0 & 3.0 & $12.8 \times 10^{-5}$ & $3.2 \times 10^{-5}$ & 0.655 & 0.987 & 0.00001 \\
\hline (G) & 2.2 & 3.5 & $8.8 \times 10^{-5}$ & $3.2 \times 10^{-3}$ & 0.629 & 0.996 & 0.0008 \\
\hline$(\mathrm{H})$ & 2.2 & 4.3 & $3.6 \times 10^{-5}$ & $3.2 \times 10^{-3}$ & 0.648 & 0.993 & 0.0042 \\
\hline
\end{tabular}

ments will obtain a null result. A new generation of upgraded conventional neutrino beams is being considered [19], and is expected to be able to probe the region $\sin ^{2} 2 \theta_{13}>0.003$, and hence measure the parameter $\theta_{13}$ if the solution lies in the upper part of the allowed $(a, b)$-plane indicated in the figure. A Neutrino Factory $\| 20$ is expected to be able to probe down to values of $\sin ^{2} 2 \theta_{13}$ as low as $O\left(10^{-4}\right)$, which will therefore cover the entire allowed $(a, b)$-plane, except for a narrow band in which $\sin ^{2} 2 \theta_{13} \rightarrow 0$ as $\sin ^{2} 2 \theta_{23}$ becomes maximal.

Figure 2 shows, once again, the viable region of parameter space consistent with the LMA solar solution, but this time with contours of constant $\Delta m_{21}^{2}$ displayed. These contours are approximately at $45^{\circ}$ in the $(a, b)$-plane, and are almost parallel to the contours of constant $\sin ^{2} 2 \theta_{13}$ shown in Fig. 1. This implies a remarkable correlation between the predicted values of $\Delta m_{21}^{2}$ and $\sin ^{2} 2 \theta_{13}$. This correlation is shown explicitly in Fig. 3 which displays, for a grid of points that span the allowed region of the $(a, b)$-parameter space, the predicted values of $\left(\Delta m_{21}^{2}, \sin ^{2} 2 \theta_{13}\right)$. The points are confined to a narrow band, with $\sin ^{2} 2 \theta_{12}$ varying across the band. Note that if the LMA solution is indeed the correct solution to explain the solar neutrino deficit observations, KamLAND [21] is expected to provide measurements of $\Delta m_{21}^{2}$ and $\sin ^{2} 2 \theta_{12}$. Hence the GUT model we are considering will be able to give a precise prediction for $\sin ^{2} 2 \theta_{13}$.

In Table 1 we have selected eight points in the LMA allowed parameter region to illustrate the neutrino oscillation parameter predictions of the GUT model. The correlations noted above are evident.

We next consider the sensitivity of the predicted oscillation parameters to the assumed values of the underlying GUT model parameters. For a grid of points in the $(a, b)$-plane, Table [1] lists the $(\Delta a / a) /\left(\Delta \sin ^{2} 2 \theta_{12} / \sin ^{2} 2 \theta_{12}\right)$, i.e., the fractional changes in the GUT scale parameter $a$ divided by the fractional changes in the predicted oscillation parameter $\sin ^{2} 2 \theta_{12}$. The values vary from -1.2 to -5.5 over the viable region of the $(a, b)$-plane. Hence, if the parameter $a$ is increased by $1 \%$, say, then the predicted value of $\sin ^{2} 2 \theta_{12}$ will typically decrease by a few percent. The corresponding sensitivity of the predicted value of $\Delta m_{21}^{2}$ to changes in $a$ is shown in Table III. Note that if the parameter $a$ is increased by $1 \%$, say, then the predicted value of $\Delta m_{21}^{2}$ increases typically by a fraction of a percent. Similar 


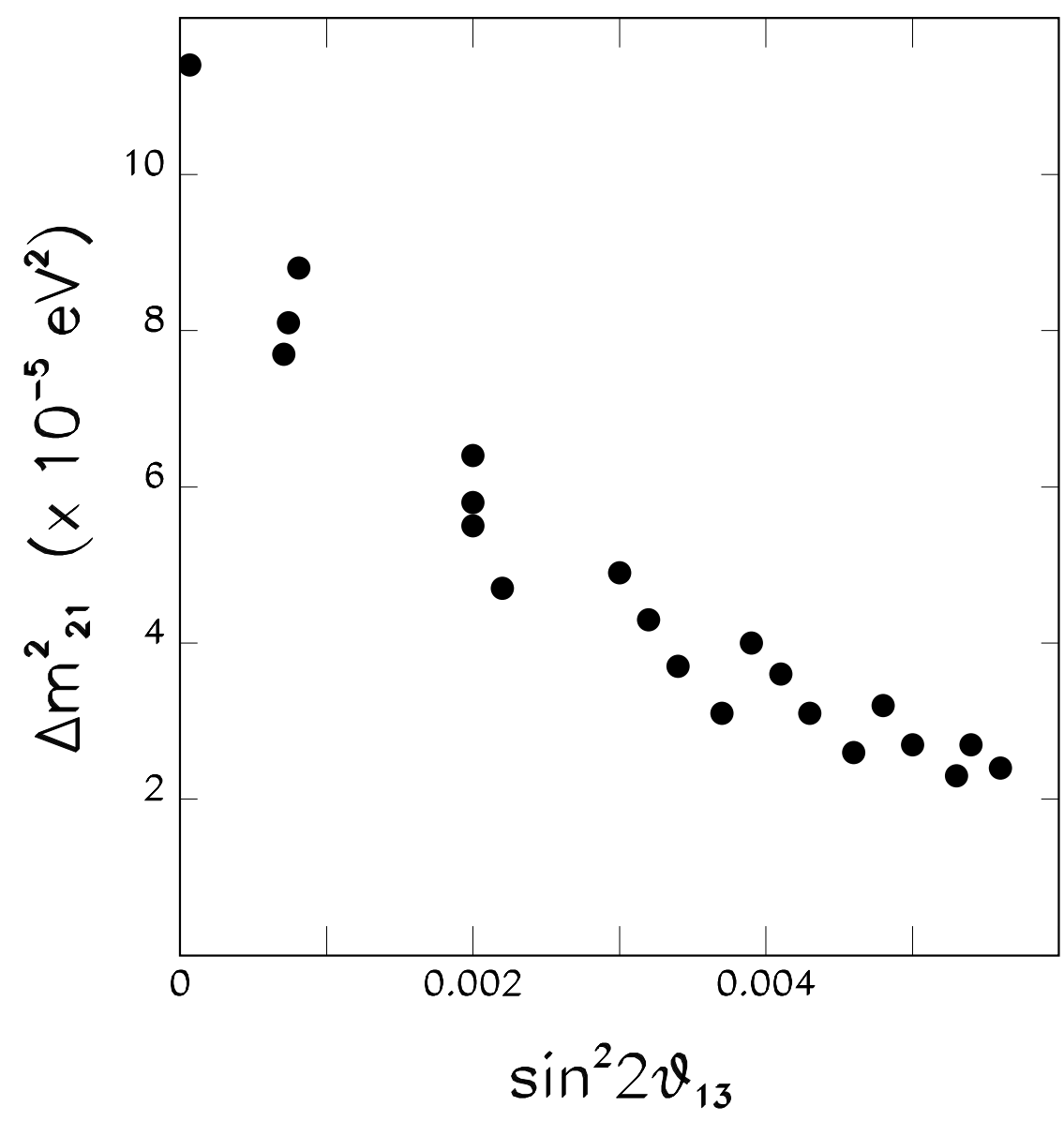

FIG. 3. Variation of $\sin ^{2} 2 \theta_{13}$ with $\Delta m_{21}^{2}$. The points plotted populate a grid which spans the viable region of the $(a, b)$ parameter space. The small spread in points across the band indicated arises from the variation in $\sin ^{2} 2 \theta_{12}$ for the points plotted.

sensitivities are expected for the predicted values of $\sin ^{2} 2 \theta_{13}$ with changes in $a$ (Table IV), or for the predicted values of $\Delta m_{21}^{2}$ (Table $\nabla$ ) or $\sin ^{2} 2 \theta_{13}$ (Table $\nabla \mathbb{1}$ ) with changes in $b$. The predicted values of $\sin ^{2} 2 \theta_{12}$ are insensitive to the value of $b$ (not shown in the tables). From these considerations we see that a precise measurement of $\sin ^{2} 2 \theta_{12}$ by KamLAND will precisely determine the GUT model parameter $a$ (for real $a$ ). A very precise measurement of either $\Delta m_{21}^{2}$ or $\sin ^{2} 2 \theta_{13}$ will then precisely determine $b$.

In summary, our examination of the simplest case ( $a$ and $b=c$ real) has revealed some striking features:

(i) A large value for $\sin ^{2} 2 \theta_{13}$ cannot be accommodated. In fact the model predicts $\sin ^{2} \theta_{13}<$ 0.01 .

(ii) The prediction for $\sin ^{2} 2 \theta_{13}$ is precise once $\Delta m_{21}^{2}$ and $\sin ^{2} 2 \theta_{12}$ are known. 
TABLE II. Fractional change in the GUT scale parameter divided by the resulting fractional change in the oscillation parameter: $(\Delta a / a) /\left(\Delta \sin ^{2} 2 \theta_{12} / \sin ^{2} 2 \theta_{12}\right)$.

\begin{tabular}{c|cccccc}
\hline \hline & & & $\mathrm{a}$ & & \\
$\mathrm{b}$ & 1.2 & 1.4 & 1.6 & 1.8 & 2.0 & 2.2 \\
\hline 4.5 & & & & & & -1.7 \\
4.0 & & -4.6 & -3.1 & -2.7 & -2.0 & -1.3 \\
3.5 & -5.5 & -3.8 & -2.4 & -2.0 & -1.4 & \\
3.0 & -4.0 & -2.6 & -1.2 & -1.3 & \\
2.5 & -2.8 & & & & \\
2.0 & & & & \\
\hline \hline
\end{tabular}

TABLE III. Fractional change in the GUT scale parameter divided by the resulting fractional change in the oscillation parameter: $(\Delta a / a) /\left(\Delta\left(\Delta m_{21}^{2}\right) / \Delta m_{21}^{2}\right)$.

\begin{tabular}{c|cccccc}
\hline \hline & & & $\mathrm{a}$ & & \\
$\mathrm{b}$ & 1.2 & 1.4 & 1.6 & 1.8 & 2.0 & 2.2 \\
\hline 4.5 & & & & & & 0.3 \\
4.0 & & 0.5 & 0.4 & 0.4 & 0.3 & 0.3 \\
3.5 & 0.5 & 0.4 & 0.4 & 0.4 & 0.3 & \\
3.0 & 0.3 & 0.2 & & 0.2 & & \\
2.5 & 0.2 & & & & & \\
2.0 & 0.0 & & & \\
\hline \hline
\end{tabular}

TABLE IV. Fractional change in the GUT scale parameter divided by the resulting fractional change in the oscillation parameter: $(\Delta a / a) /\left(\Delta \sin ^{2} 2 \theta_{13} / \sin ^{2} 2 \theta_{13}\right)$.

\begin{tabular}{c|cccccc}
\hline \hline & & & $\mathrm{a}$ & & \\
$\mathrm{b}$ & 1.2 & 1.4 & 1.6 & 1.8 & 2.0 & 2.2 \\
\hline 4.5 & & & & & -0.7 & -0.6 \\
4.0 & & -0.8 & -0.5 & -0.7 & -0.4 & -0.3 \\
3.5 & -0.6 & -0.4 & -0.2 & -0.1 & -0.2 & -0.1 \\
3.0 & -0.1 & -0.1 & & \\
2.5 & -0.2 & & & & \\
2.0 & & & & & \\
\hline \hline
\end{tabular}


TABLE V. Fractional change in the GUT scale parameter divided by the resulting fractional change in the oscillation parameter: $(\Delta b / b) /\left(\Delta\left(\Delta m_{21}^{2}\right) / \Delta m_{21}^{2}\right)$.

\begin{tabular}{c|cccccc}
\hline \hline & & & $\mathrm{a}$ & & \\
$\mathrm{b}$ & 1.2 & 1.4 & 1.6 & 1.8 & 2.0 & -0.2 \\
\hline 4.5 & & & & & & \\
4.0 & & & -0.1 & -0.2 & -0.2 & \\
3.5 & -0.2 & -0.2 & -0.2 & & \\
3.0 & -0.2 & -0.4 & & & \\
2.5 & -0.4 & & & & \\
2.0 & - & & & \\
\hline \hline
\end{tabular}

TABLE VI. Fractional change in the GUT scale parameter divided by the resulting fractional change in the oscillation parameter: $(\Delta b / b) /\left(\Delta \sin ^{2} 2 \theta_{13} / \sin ^{2} 2 \theta_{13}\right)$.

\begin{tabular}{c|cccccc}
\hline \hline & & & $\mathrm{a}$ & & \\
$\mathrm{b}$ & 1.2 & 1.4 & 1.6 & 1.8 & 2.0 & 2.2 \\
\hline 4.5 & & & & 0.4 & 0.3 \\
4.0 & & 0.4 & 0.3 & 0.4 & 0.3 & 0.2 \\
3.5 & 0.3 & 0.3 & 0.2 & 0.05 & 0.1 & 0.05 \\
3.0 & 0.2 & 0.05 & & & & \\
2.5 & & & & & \\
2.0 & & & & & \\
\hline \hline
\end{tabular}

\section{B. Parameter Choice: $b=c$ Real with $a$ Complex}

We have seen from the example presented in Sec. III that the CP phase, $\delta_{C P}$, turns out to be very small, since both the Dirac neutrino matrix $N$ and the right-handed Majorana matrix $M_{R}$ are real, while only the charged lepton matrix $L$ is complex and results in a small complex contribution to $U_{M N S}$. But with two Higgs lepton-violating singlets contributing to $M_{R}$, one can introduce an additional complex phase $\phi^{\prime}$ into $M_{R}$. In discussing CP violation, we shall identify

$$
a \equiv b-a^{\prime} e^{i \phi^{\prime}}, \text { with } b=c
$$

where $b$ is real and arises from the first Higgs singlet which contributes to all nine matrix elements of $M_{R}$, while $a^{\prime}$ can be complex and arises from the second Higgs singlet which contributes to only the 13 and 31 elements. Any observable CP violation in the lepton sector with its phase $\delta_{C P}$ is then controlled by $\phi^{\prime}$ and the phase $\phi$ appearing in the charged lepton matrix $L$ in Eq. (6). The viable region of parameter space shown in Fig. [1 and 2 is not significantly changed. To understand the predictions in detail, we again choose the eight specific points in parameter space listed earlier in Table If. For each point, the predictions for $\sin ^{2} 2 \theta_{12}, \sin ^{2} 2 \theta_{23}, \sin ^{2} 2 \theta_{13}$, and $\delta_{C P}$ are listed as functions of $\phi^{\prime}$ in Tables VII - XIV. The predicted observable $\delta_{C P}$, as well as the Majorana phase $\chi_{1}$, are shown 
for each point as functions of $\phi^{\prime}$ in Figs. 4and 5. Only the range $\left|\phi^{\prime}\right|<75^{\circ}$ within the dashed lines in these figures is consistent with the present lower limit on $\sin ^{2} 2 \theta_{23}$. Note that

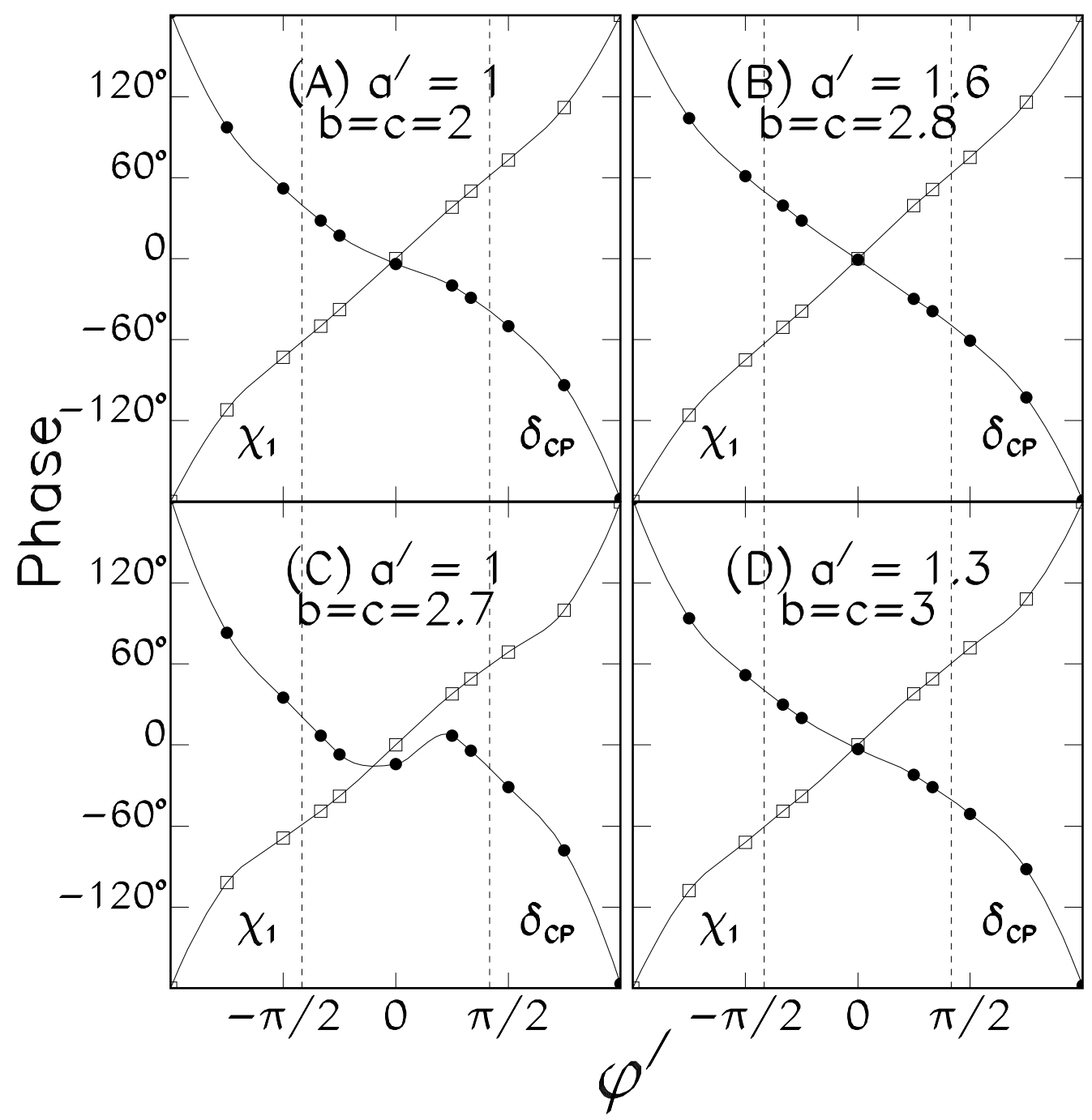

FIG. 4. The observable CP phase $\delta_{C P}$ and Majorana phase $\chi_{1}$ are shown as functions of the GUT phase parameter $\phi^{\prime}$ for the first four of the eight points in parameter space that are listed in Table f. The ranges of $\phi^{\prime}$ of interest lie between the dashed lines.

when $\phi^{\prime}=0$, corresponding to the maximum values predicted for $\sin ^{2} 2 \theta_{23}$, the predictions for $\delta_{C P}$ are typically a few degrees except for cases $(\mathrm{C})$ and $(\mathrm{F})$ for which $\delta_{C P}=-14^{\circ}$ and $-50^{\circ}$, respectively. The peculiar behavior for these two special cases arises because $\sin ^{2} 2 \theta_{23}$ becomes maximal and crosses from the dark side $\left(\tan \theta_{23}>1\right)$ into the light side $\left(\tan \theta_{23}<1\right)$ and back into the dark side as $\phi^{\prime}$ goes through $0^{\circ}$. The predictions for $\chi_{1}$ and $\chi_{2}$, on the other hand, are smoothly varying in all cases. Since $\Delta m_{21}^{2}$ is on the high side of the allowed region for cases (C) and (F), and somewhat disfavored by other recent analyses 
[10], it appears that the GUT model under consideration predicts that leptonic CP violation will be small for the near maximal values of $\sin ^{2} 2 \theta_{23}$ and more generally that $\left|\delta_{C P}\right|<50^{\circ}$.

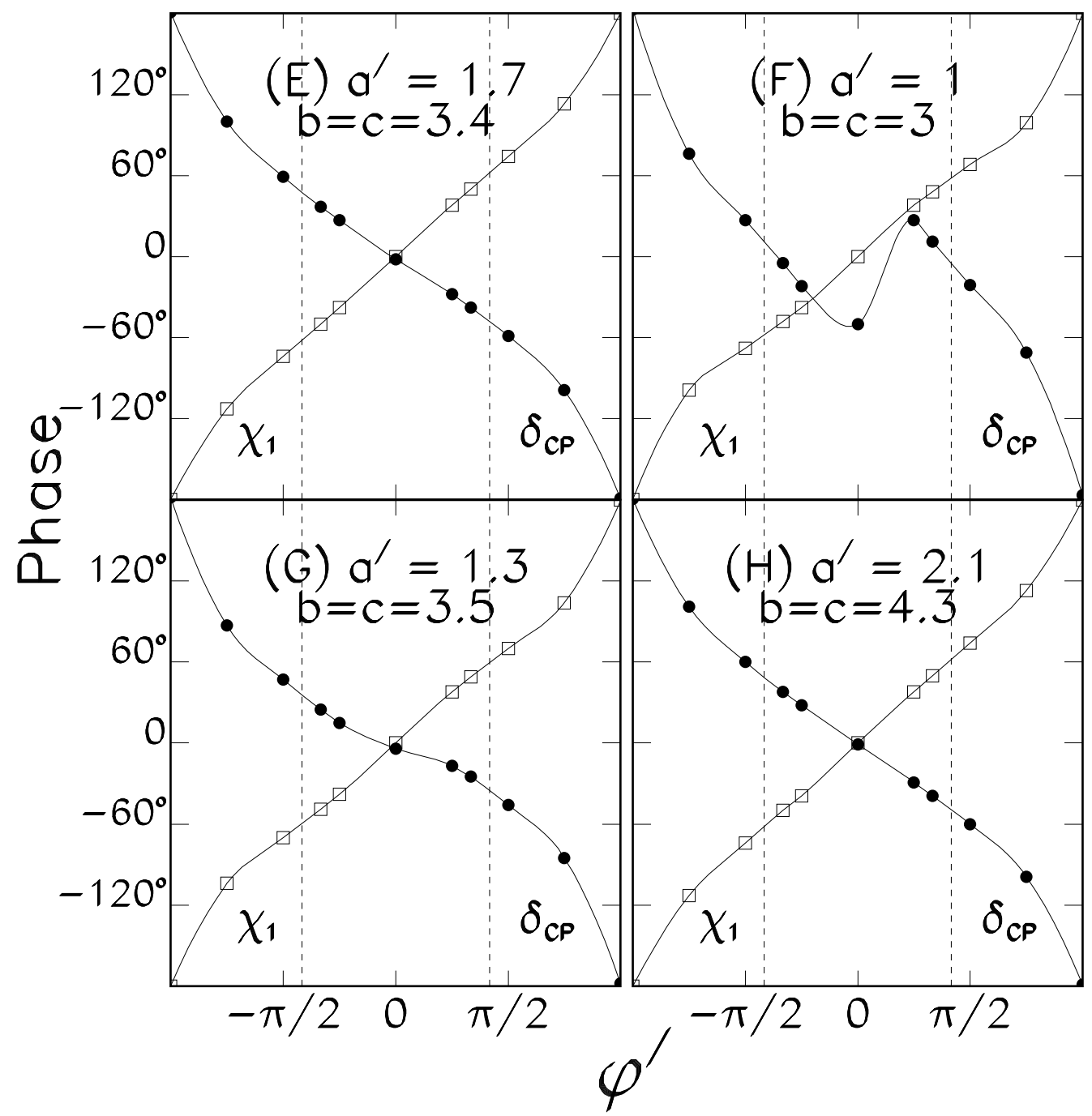

FIG. 5. The observable CP phase $\delta_{C P}$ and Majorana phase $\chi_{1}$ are shown as functions of the GUT phase parameter $\phi^{\prime}$ for the second four of the eight points in parameter space that are listed in Table 1. The ranges of $\phi^{\prime}$ of interest lie between the dashed lines.

Next consider the predictions for the mixing angles, $\sin ^{2} 2 \theta_{12}$ and $\sin ^{2} 2 \theta_{23}$, which are shown for the 8 points in parameter space in Figs. 6 and 7 . These figures show the predictions as functions of $\phi^{\prime}$. Within the viable region of parameter space corresponding to $\sin ^{2} 2 \theta_{23}>0.89$, the permitted values of $\sin ^{2} 2 \theta_{12}$ are restricted for each point in $\left(a^{\prime}, b\right)$-space. A $10 \%$ measurement of $\sin ^{2} 2 \theta_{12}$ by the KamLAND experiment, combined with a few percent measurement of $\sin ^{2} 2 \theta_{23}$ by MINOS and the CNGS experiments would enable significant regions of the GUT model parameter space to be excluded. A $1 \%$ measurement of $\sin ^{2} 2 \theta_{23}$ 
at a Neutrino Factory would provide a stringent test of the GUT model.

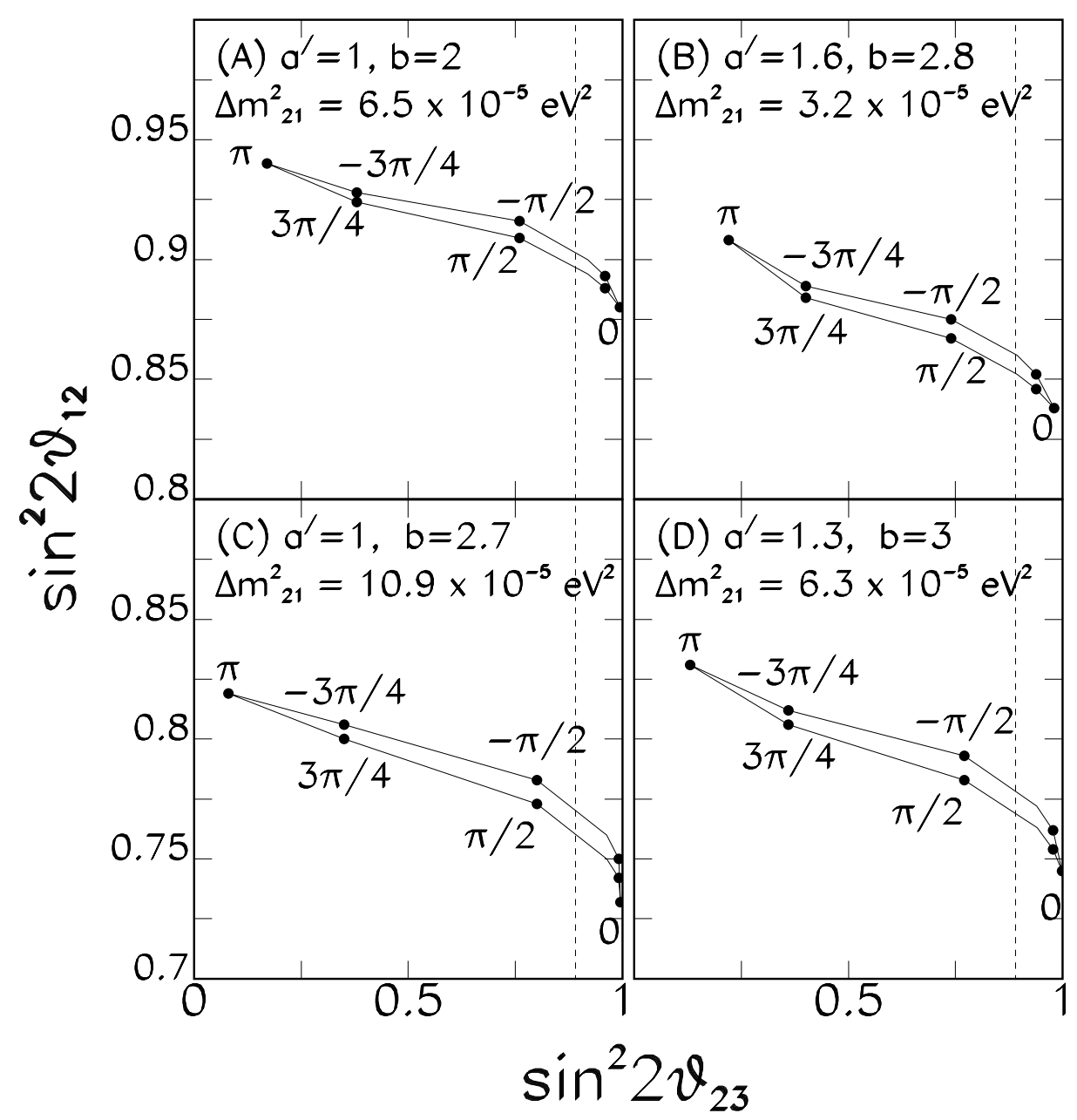

FIG. 6. The predicted value of $\sin ^{2} 2 \theta_{12}$ shown as a function of the predicted $\sin ^{2} 2 \theta_{23}$ for the first four of the eight points in parameter space that are listed in Table $\mathbb{1}$. The values of $\delta_{C P}$ vary around the contour of solutions and are indicated at points corresponding to $\phi^{\prime}=0, \pm \pi / 4, \pm \pi / 2, \pm 3 \pi / 4$, and $\pi$. The viable region in $\sin ^{2} 2 \theta_{23}$ lies between 0.89 and 1.0.

\section{Parameter Choice: $a \neq b \neq c$}

The more general GUT model case with $a \neq b \neq c$ would arise if three Higgs VEVs breaking lepton number were to contribute to the right-handed Majorana mass matrix. This complication is much more difficult to analyse and is not studied here. The two simplified cases we have studied appear sufficient to present a realistic picture of neutrino oscillations. 


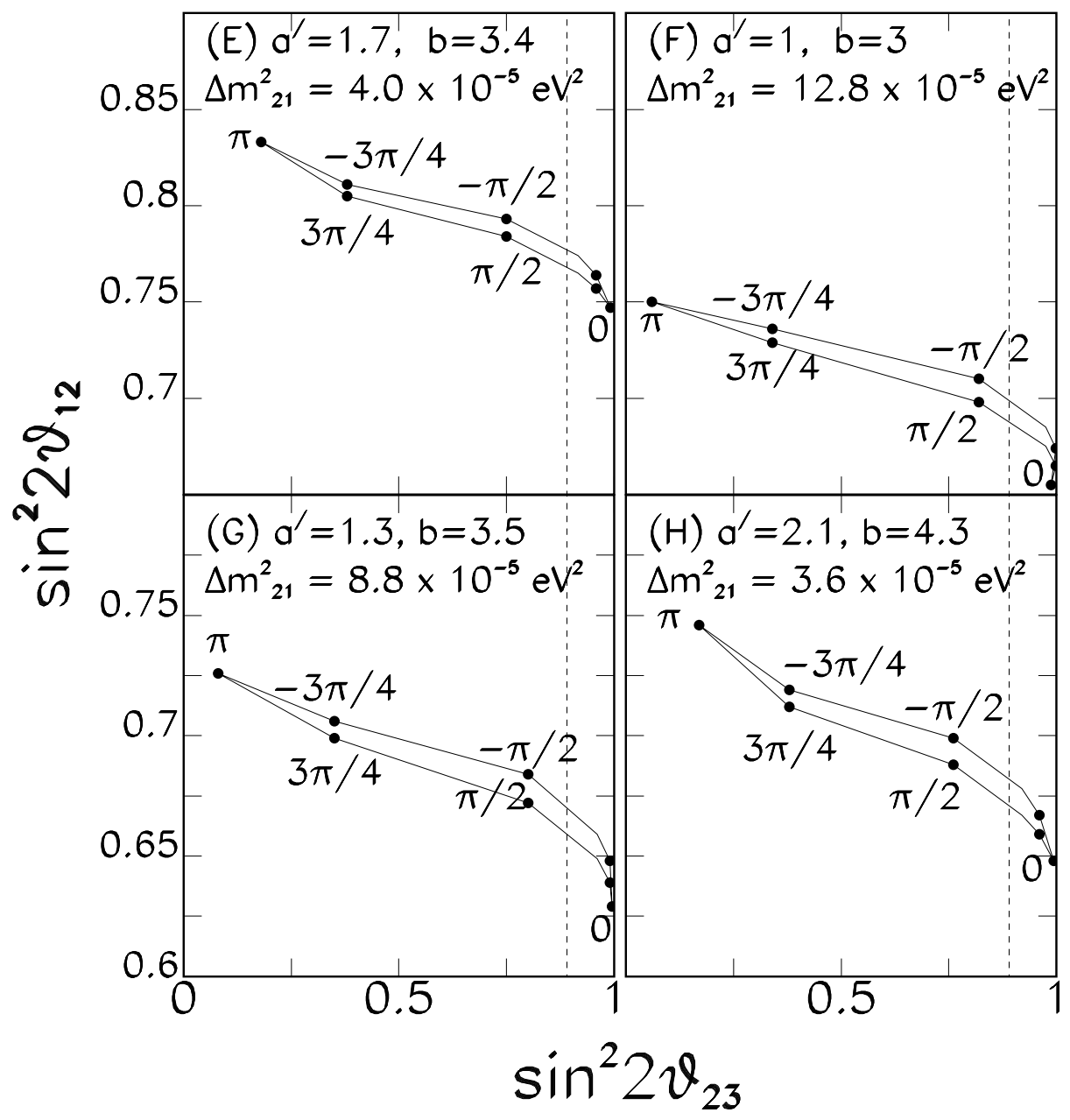

FIG. 7. The predicted value of $\sin ^{2} 2 \theta_{12}$ shown as a function of the predicted $\sin ^{2} 2 \theta_{23}$ for the second four of the eight points in parameter space that are listed in Table $\mathbb{1}$. The values of $\delta_{C P}$ vary around the contour of solutions and are indicated at points corresponding to $\phi^{\prime}=0, \pm \pi / 4, \pm \pi / 2, \pm 3 \pi / 4$, and $\pi$. 


\section{CONCLUSIONS}

Within the framework of an $S O(10)$ GUT model developed by Albright and Barr that can accommodate both the atmospheric and LMA solar neutrino mixing solutions, we have presented explicit predictions for $\sin ^{2} 2 \theta_{13}, \sin ^{2} 2 \theta_{12}, \sin ^{2} 2 \theta_{23}$, and $\Delta m_{21}^{2}$. Precise measurements of $\sin ^{2} 2 \theta_{12}$ and $\Delta m_{21}^{2}$ by KamLAND can be used to precisely determine the GUT parameters $a$ (with $a$ real) and $b$. We find that the model can then be tested with precision neutrino oscillation measurements of $\sin ^{2} 2 \theta_{23}, \sin ^{2} 2 \theta_{13}$, and the leptonic CP phase $\delta^{\prime}$ at Neutrino Superbeams and Neutrino Factories.

Over the entire region of viable GUT model parameter space, the value of $\sin ^{2} 2 \theta_{13}$ is predicted to be less than 0.01. If this is the case, $\nu_{\mu} \rightarrow \nu_{e}$ oscillations will not be observed by the MINOS or CNGS experiments. Over half of the viable parameter space, the predicted $\sin ^{2} 2 \theta_{13}$ exceeds 0.003 , and $\nu_{\mu} \rightarrow \nu_{e}$ oscillations would be expected to be observed at Neutrino Superbeams. The remaining half of the parameter space would be probed at a Neutrino Factory, except a small region for which $\sin ^{2} 2 \theta_{13}<0.0001$. The GUT model predicts a striking correlation between $\Delta m_{21}^{2}$ and $\sin ^{2} 2 \theta_{13}$. Once $\Delta m_{21}^{2}$ is measured by KamLAND with a precision of a few percent, the model will predict $\sin ^{2} 2 \theta_{13}$ with a precision of a few percent. A precise test of the model with this level of precision will require a Neutrino Factory.

In the more general version of the GUT model in which $a$ is complex, the absolute observable CP phase $\left|\delta_{C P}\right|$ is at most $\sim 50^{\circ}$ over almost the entire viable parameter space. For the maximal atmospheric neutrino mixing region, $\delta_{C P}$ is typically very small, with exceptions noted earlier for the largest values of $\Delta m_{21}^{2}$ presently allowed. The predicted $\left\langle m_{\beta \beta}\right\rangle$ is at most a few times $10^{-4} \mathrm{eV}$, too small for neutrinoless double beta decay to be observed by the next generation experiments.

Finally, a general conclusion from the study of the predictions of one specific GUT model is that, if the LMA solar solution is confirmed, very precise measurements of all the oscillation parameters are important to test the theory and determine the associated parameters. We will need a Neutrino Factory.

The initial preparation of this manuscript was carried out at the Snowmass 2001 Workshop on the Future of Particle Physics. One of us (CHA) thanks Stephen Barr for several discussions on the complex extension of the right-handed Majorana neutrino mass matrix that was developed in collaboration with him for the LMA solution. 


\section{REFERENCES}

[1] Super-Kamiokande Collab., S. Fukuda et al., Phys. Rev. Lett. 85, 3999 (2000).

[2] CHOOZ Collab., M. Apollonio et al., Phys. Lett. B 420, 397 (1998).

[3] K. Lande et al., Astrophys. J. 496, 505 (1998).

[4] SNO Collab., Q.R. Ahmad et al., nucl-ex/0106015.

[5] J.N. Bahcall, M.H. Pinsonneault, and S. Basu, Astrophys. J. 555, 990 (2001).

[6] Super-Kamiokande Collab., S. Fukuda et al., Phys. Rev. Lett. 86, 5651 (2001); ibid. 86, 5656 (2001).

[7] L. Wolfenstein, Phys. Rev. D 17, 2369 (1978); S.P. Mikheyev and A. Yu. Smirnov, Yad. Fiz. 42, 1441 (1985), [Sov. J. Nucl. Phys. 42, 913 (1985)].

[8] M. Gell-Mann, P. Ramond, and R. Slansky, Report No. CALT-68-709: Supergravity (North Holland, Amsterdam, 1979); T. Yanagida, in Proceedings of the Workshop on Unified Theory and Baryon Number of the Universe, KEK, Japan, 1979, unpublished.

[9] Z. Maki, M. Nakagawa, and S. Sakata, Prog. Theor. Phys. 28, 870 (1962).

[10] G.L. Fogli, E. Lisi, D. Montanino, and A. Palazzo, Phys. Rev. D 62, 113003 (2000); M.C. Gonzalez-Garcia, M. Maltoni, C. Pena-Garay, and J.W.F. Valle, Phys. Rev. D 63, 033005 (2001); J.N. Bahcall, P.I. Krastev, and A.Yu. Smirnov, hep-ph/0103179.

[11] C.H. Albright and S.M. Barr, Phys. Rev. Lett. 85, 244 (2000); Phys. Rev. D 62, 093008 (2000); Phys. Rev. D 64, 073010 (2001).

[12] S.M. Barr and S. Raby, Phys. Rev. Lett. 79, 4748 (1997).

[13] H. Georgi and C. Jarlskog, Phys. Lett. B 86, 297 (1979).

[14] C.D. Froggatt and H.B. Nielsen, Nucl. Phys. B 147, 277 (1979).

[15] C.H. Albright, K.S. Babu, and S.M. Barr, Phys. Rev. Lett. 81, 1167 (1998).

[16] C. Jarlskog, Phys. Rev. Lett. 55, 1039 (1985).

[17] S.G. Wojcicki, Nucl. Phys. Proc. Suppl. 91, 216 (2001).

[18] A. Rubbia, Nucl. Phys. Proc. Suppl. 91, 223 (2000).

[19] V. Barger, S. Geer, R. Raja, and K. Whisnant, Phys. Rev. D 63, 113011 (2001).

[20] S. Geer, Phys. Rev. D 57, 6989 (1998).

[21] KamLAND Collab., A. Piepke et al., Nucl. Phys. Proc. Suppl. 91, 99 (2001). 
TABLE VII. Oscillation Parameters for Point (A)

(A) $M_{R}$ Model Parameters: $\quad a^{\prime}=1.0, b=c=2.0, \Lambda_{R}=2.5 \times 10^{14} \mathrm{GeV}$

Predictions: $\Delta m_{21}^{2}=6.5 \times 10^{-5} \mathrm{eV}^{2}, \quad \Delta m_{32}^{2}=3.2 \times 10^{-3} \mathrm{eV}^{2}$

\begin{tabular}{rcccrrr}
\multicolumn{1}{c}{$\phi^{\prime}$} & $\sin ^{2} 2 \theta_{12}$ & $\sin ^{2} 2 \theta_{23}$ & $\sin ^{2} 2 \theta_{13}$ & $\delta_{C P}$ & \multicolumn{1}{c}{$\chi_{1}$} & \multicolumn{1}{c}{$\chi_{2}$} \\
\hline \hline$-\pi$ & 0.940 & 0.17 & 0.0033 & $182^{\circ}$ & $-180^{\circ}$ & $-180^{\circ}$ \\
$-3 \pi / 4$ & 0.928 & 0.38 & 0.0031 & $97^{\circ}$ & $-112^{\circ}$ & $-109^{\circ}$ \\
$-\pi / 2$ & 0.916 & 0.76 & 0.0022 & $52^{\circ}$ & $-73^{\circ}$ & $-69^{\circ}$ \\
\hline$-\pi / 3$ & 0.900 & 0.919 & 0.0015 & $28^{\circ}$ & $-50^{\circ}$ & $-47^{\circ}$ \\
$-\pi / 4$ & 0.893 & 0.960 & 0.0013 & $17^{\circ}$ & $-38^{\circ}$ & $-35^{\circ}$ \\
0 & 0.880 & 0.994 & 0.0008 & $-4^{\circ}$ & $0^{\circ}$ & $0^{\circ}$ \\
$\pi / 4$ & 0.888 & 0.960 & 0.0010 & $-20^{\circ}$ & $38^{\circ}$ & $35^{\circ}$ \\
$\pi / 3$ & 0.894 & 0.919 & 0.0013 & $-29^{\circ}$ & $50^{\circ}$ & $47^{\circ}$ \\
\hline$\pi / 2$ & 0.909 & 0.76 & 0.0019 & $-50^{\circ}$ & $73^{\circ}$ & $69^{\circ}$ \\
$3 \pi / 4$ & 0.924 & 0.38 & 0.0028 & $-94^{\circ}$ & $112^{\circ}$ & $109^{\circ}$ \\
$\pi$ & 0.940 & 0.17 & 0.0033 & $-178^{\circ}$ & $180^{\circ}$ & $180^{\circ}$ \\
\hline \hline
\end{tabular}

TABLE VIII. Oscillation Parameters for Point (B)

(B) $M_{R}$ Model Parameters: $\quad a^{\prime}=1.6, b=c=2.8, \Lambda_{R}=2.4 \times 10^{14} \mathrm{GeV}$

Predictions: $\quad \Delta m_{21}^{2}=3.2 \times 10^{-5} \mathrm{eV}^{2}, \quad \Delta m_{32}^{2}=3.2 \times 10^{-3} \mathrm{eV}^{2}$

\begin{tabular}{rccrrrr}
\multicolumn{1}{c}{$\phi^{\prime}$} & $\sin ^{2} 2 \theta_{12}$ & $\sin ^{2} 2 \theta_{23}$ & $\sin ^{2} 2 \theta_{13}$ & $\delta_{C P}$ & \multicolumn{1}{c}{$\chi_{1}$} & $\chi_{2}$ \\
\hline \hline$-\pi$ & 0.908 & 0.22 & 0.0077 & $181^{\circ}$ & $-180^{\circ}$ & $-180^{\circ}$ \\
$-3 \pi / 4$ & 0.889 & 0.40 & 0.0073 & $104^{\circ}$ & $-116^{\circ}$ & $-114^{\circ}$ \\
$-\pi / 2$ & 0.875 & 0.74 & 0.0060 & $61^{\circ}$ & $-75^{\circ}$ & $-72^{\circ}$ \\
\hline$-\pi / 3$ & 0.860 & 0.894 & 0.0049 & $39^{\circ}$ & $-51^{\circ}$ & $-48^{\circ}$ \\
$-\pi / 4$ & 0.852 & 0.938 & 0.0045 & $28^{\circ}$ & $-39^{\circ}$ & $-36^{\circ}$ \\
0 & 0.838 & 0.980 & 0.0038 & $-1^{\circ}$ & $0^{\circ}$ & $0^{\circ}$ \\
$\pi / 4$ & 0.846 & 0.938 & 0.0042 & $-30^{\circ}$ & $39^{\circ}$ & $36^{\circ}$ \\
$\pi / 3$ & 0.852 & 0.894 & 0.0045 & $-39^{\circ}$ & $51^{\circ}$ & $48^{\circ}$ \\
\hline$\pi / 2$ & 0.867 & 0.74 & 0.0055 & $-61^{\circ}$ & $75^{\circ}$ & $72^{\circ}$ \\
$3 \pi / 4$ & 0.884 & 0.40 & 0.0069 & $-103^{\circ}$ & $116^{\circ}$ & $114^{\circ}$ \\
$\pi$ & 0.908 & 0.22 & 0.0077 & $-179^{\circ}$ & $180^{\circ}$ & $180^{\circ}$ \\
\hline \hline
\end{tabular}


TABLE IX. Oscillation Parameters for Point (C)

(C) $M_{R}$ Model Parameters: $\quad a^{\prime}=1.0, b=c=2.7, \Lambda_{R}=2.5 \times 10^{14} \mathrm{GeV}$

Predictions: $\quad \Delta m_{21}^{2}=10.9 \times 10^{-5} \mathrm{eV}^{2}, \quad \Delta m_{32}^{2}=3.2 \times 10^{-3} \mathrm{eV}^{2}$

\begin{tabular}{rccrrrr}
\multicolumn{1}{c}{$\phi^{\prime}$} & $\sin ^{2} 2 \theta_{12}$ & $\sin ^{2} 2 \theta_{23}$ & $\sin ^{2} 2 \theta_{13}$ & $\delta_{C P}$ & \multicolumn{1}{c}{$\chi_{1}$} & $\chi_{2}$ \\
\hline \hline$-\pi$ & 0.819 & 0.08 & 0.0021 & $183^{\circ}$ & $-180^{\circ}$ & $-180^{\circ}$ \\
$-3 \pi / 4$ & 0.806 & 0.35 & 0.0019 & $83^{\circ}$ & $-102^{\circ}$ & $-100^{\circ}$ \\
$-\pi / 2$ & 0.783 & 0.80 & 0.0012 & $35^{\circ}$ & $-69^{\circ}$ & $-66^{\circ}$ \\
\hline$-\pi / 3$ & 0.760 & 0.963 & 0.0007 & $7^{\circ}$ & $-49^{\circ}$ & $-45^{\circ}$ \\
$-\pi / 4$ & 0.750 & 0.992 & 0.0005 & $-7^{\circ}$ & $-38^{\circ}$ & $-35^{\circ}$ \\
0 & 0.732 & 0.996 & 0.0001 & $-14^{\circ}$ & $0^{\circ}$ & $0^{\circ}$ \\
$\pi / 4$ & 0.742 & 0.992 & 0.0003 & $7^{\circ}$ & $38^{\circ}$ & $35^{\circ}$ \\
$\pi / 3$ & 0.750 & 0.963 & 0.0005 & $-4^{\circ}$ & $49^{\circ}$ & $45^{\circ}$ \\
\hline$\pi / 2$ & 0.773 & 0.80 & 0.0010 & $-31^{\circ}$ & $69^{\circ}$ & $66^{\circ}$ \\
$3 \pi / 4$ & 0.800 & 0.35 & 0.0017 & $-78^{\circ}$ & $100^{\circ}$ & $102^{\circ}$ \\
$\pi$ & 0.819 & 0.08 & 0.0021 & $-177^{\circ}$ & $180^{\circ}$ & $180^{\circ}$ \\
\hline \hline
\end{tabular}

TABLE X. Oscillation Parameters for Point (D)

\section{(D) $M_{R}$ Model Parameters: $\quad a^{\prime}=1.3, b=c=3.0, \Lambda_{R}=2.5 \times 10^{14} \mathrm{GeV}$}

Predictions: $\Delta m_{21}^{2}=6.3 \times 10^{-5} \mathrm{eV}^{2}, \quad \Delta m_{32}^{2}=3.2 \times 10^{-3} \mathrm{eV}^{2}$

\begin{tabular}{rcccrrr}
\multicolumn{1}{c}{$\phi^{\prime}$} & $\sin ^{2} 2 \theta_{12}$ & $\sin ^{2} 2 \theta_{23}$ & $\sin ^{2} 2 \theta_{13}$ & $\delta_{C P}$ & \multicolumn{1}{c}{$\chi_{1}$} & \multicolumn{1}{c}{$\chi_{2}$} \\
\hline \hline$-\pi$ & 0.831 & 0.13 & 0.0049 & $182^{\circ}$ & $-180^{\circ}$ & $-180^{\circ}$ \\
$-3 \pi / 4$ & 0.812 & 0.36 & 0.0045 & $94^{\circ}$ & $-108^{\circ}$ & $-106^{\circ}$ \\
$-\pi / 2$ & 0.793 & 0.77 & 0.0033 & $52^{\circ}$ & $-72^{\circ}$ & $-68^{\circ}$ \\
\hline$-\pi / 3$ & 0.772 & 0.939 & 0.0024 & $30^{\circ}$ & $-49^{\circ}$ & $-46^{\circ}$ \\
$-\pi / 4$ & 0.762 & 0.977 & 0.0020 & $20^{\circ}$ & $-38^{\circ}$ & $-35^{\circ}$ \\
0 & 0.745 & 0.9991 & 0.0014 & $-3^{\circ}$ & $0^{\circ}$ & $0^{\circ}$ \\
$\pi / 4$ & 0.754 & 0.977 & 0.0017 & $-22^{\circ}$ & $38^{\circ}$ & $35^{\circ}$ \\
$\pi / 3$ & 0.763 & 0.940 & 0.0021 & $-31^{\circ}$ & $49^{\circ}$ & $46^{\circ}$ \\
\hline$\pi / 2$ & 0.783 & 0.77 & 0.0029 & $-51^{\circ}$ & $72^{\circ}$ & $68^{\circ}$ \\
$3 \pi / 4$ & 0.806 & 0.36 & 0.0042 & $-92^{\circ}$ & $108^{\circ}$ & $106^{\circ}$ \\
$\pi$ & 0.831 & 0.13 & 0.0049 & $-178^{\circ}$ & $180^{\circ}$ & $180^{\circ}$ \\
\hline \hline
\end{tabular}


TABLE XI. Oscillation Parameters for Point (E)

(E) $M_{R}$ Model Parameters: $\quad a^{\prime}=1.7, b=c=3.4, \Lambda_{R}=2.5 \times 10^{14} \mathrm{GeV}$

Predictions: $\Delta m_{21}^{2}=4.0 \times 10^{-5} \mathrm{eV}^{2}, \quad \Delta m_{32}^{2}=3.2 \times 10^{-3} \mathrm{eV}^{2}$

\begin{tabular}{rccrrrr}
\multicolumn{1}{c}{$\phi^{\prime}$} & $\sin ^{2} 2 \theta_{12}$ & $\sin ^{2} 2 \theta_{23}$ & $\sin ^{2} 2 \theta_{13}$ & $\delta_{C P}$ & \multicolumn{1}{c}{$\chi_{1}$} & $\chi_{2}$ \\
\hline \hline$-\pi$ & 0.833 & 0.18 & 0.0076 & $181^{\circ}$ & $-180^{\circ}$ & $-180^{\circ}$ \\
$-3 \pi / 4$ & 0.811 & 0.38 & 0.0071 & $100^{\circ}$ & $-113^{\circ}$ & $-111^{\circ}$ \\
$-\pi / 2$ & 0.793 & 0.75 & 0.0057 & $59^{\circ}$ & $-74^{\circ}$ & $-70^{\circ}$ \\
\hline$-\pi / 3$ & 0.774 & 0.916 & 0.0046 & $37^{\circ}$ & $-50^{\circ}$ & $-47^{\circ}$ \\
$-\pi / 4$ & 0.764 & 0.958 & 0.0041 & $27^{\circ}$ & $-38^{\circ}$ & $-36^{\circ}$ \\
0 & 0.747 & 0.992 & 0.0033 & $-2^{\circ}$ & $0^{\circ}$ & $0^{\circ}$ \\
$\pi / 4$ & 0.757 & 0.958 & 0.0038 & $-28^{\circ}$ & $38^{\circ}$ & $36^{\circ}$ \\
$\pi / 3$ & 0.765 & 0.916 & 0.0042 & $-38^{\circ}$ & $50^{\circ}$ & $47^{\circ}$ \\
\hline$\pi / 2$ & 0.784 & 0.75 & 0.0052 & $-59^{\circ}$ & $74^{\circ}$ & $70^{\circ}$ \\
$3 \pi / 4$ & 0.805 & 0.38 & 0.0068 & $-99^{\circ}$ & $113^{\circ}$ & $111^{\circ}$ \\
$\pi$ & 0.833 & 0.18 & 0.0076 & $-179^{\circ}$ & $180^{\circ}$ & $180^{\circ}$ \\
\hline \hline
\end{tabular}

TABLE XII. Oscillation Parameters for Point (F)

(F) $M_{R}$ Model Parameters: $\quad a^{\prime}=1.0, b=c=3.0, \Lambda_{R}=2.6 \times 10^{14} \mathrm{GeV}$

Predictions: $\quad \Delta m_{21}^{2}=12.8 \times 10^{-5} \mathrm{eV}^{2}, \quad \Delta m_{32}^{2}=3.2 \times 10^{-3} \mathrm{eV}^{2}$

\begin{tabular}{rcccrrr}
\multicolumn{1}{c}{$\phi^{\prime}$} & $\sin ^{2} 2 \theta_{12}$ & $\sin ^{2} 2 \theta_{23}$ & $\sin ^{2} 2 \theta_{13}$ & $\delta_{C P}$ & \multicolumn{1}{c}{$\chi_{1}$} & \multicolumn{1}{c}{$\chi_{2}$} \\
\hline \hline$-\pi$ & 0.750 & 0.06 & 0.0018 & $183^{\circ}$ & $-180^{\circ}$ & $-180^{\circ}$ \\
$-3 \pi / 4$ & 0.736 & 0.34 & 0.0016 & $76^{\circ}$ & $-99^{\circ}$ & $-96^{\circ}$ \\
$-\pi / 2$ & 0.710 & 0.82 & 0.0010 & $27^{\circ}$ & $-68^{\circ}$ & $-65^{\circ}$ \\
\hline$-\pi / 3$ & 0.685 & 0.975 & 0.0005 & $-5^{\circ}$ & $-48^{\circ}$ & $-45^{\circ}$ \\
$-\pi / 4$ & 0.674 & 0.998 & 0.0003 & $-22^{\circ}$ & $-38^{\circ}$ & $-35^{\circ}$ \\
0 & 0.655 & 0.987 & 0.00001 & $-50^{\circ}$ & $0^{\circ}$ & $0^{\circ}$ \\
$\pi / 4$ & 0.665 & 0.998 & 0.0002 & $27^{\circ}$ & $38^{\circ}$ & $35^{\circ}$ \\
$\pi / 3$ & 0.675 & 0.975 & 0.0004 & $11^{\circ}$ & $48^{\circ}$ & $45^{\circ}$ \\
\hline$\pi / 2$ & 0.698 & 0.82 & 0.0008 & $-21^{\circ}$ & $68^{\circ}$ & $65^{\circ}$ \\
$3 \pi / 4$ & 0.729 & 0.34 & 0.0014 & $-71^{\circ}$ & $99^{\circ}$ & $96^{\circ}$ \\
$\pi$ & 0.750 & 0.06 & 0.0018 & $-177^{\circ}$ & $180^{\circ}$ & $180^{\circ}$ \\
\hline \hline
\end{tabular}


TABLE XIII. Oscillation Parameters for Point (G)

(G) $M_{R}$ Model Parameters: $a^{\prime}=1.3, b=c=3.5, \Lambda_{R}=2.4 \times 10^{14} \mathrm{GeV}$

Predictions: $\Delta m_{21}^{2}=8.8 \times 10^{-5} \mathrm{eV}^{2}, \quad \Delta m_{32}^{2}=3.2 \times 10^{-3} \mathrm{eV}^{2}$

\begin{tabular}{rccrrrr}
\multicolumn{1}{c}{$\phi^{\prime}$} & $\sin ^{2} 2 \theta_{12}$ & $\sin ^{2} 2 \theta_{23}$ & $\sin ^{2} 2 \theta_{13}$ & $\delta_{C P}$ & \multicolumn{1}{c}{$\chi_{1}$} & \multicolumn{1}{c}{$\chi_{2}$} \\
\hline \hline$-\pi$ & 0.726 & 0.08 & 0.0043 & $182^{\circ}$ & $-180^{\circ}$ & $-180^{\circ}$ \\
$-3 \pi / 4$ & 0.706 & 0.35 & 0.0039 & $87^{\circ}$ & $-104^{\circ}$ & $-101^{\circ}$ \\
$-\pi / 2$ & 0.684 & 0.80 & 0.0027 & $47^{\circ}$ & $-70^{\circ}$ & $-66^{\circ}$ \\
\hline$-\pi / 3$ & 0.659 & 0.961 & 0.0019 & $25^{\circ}$ & $-49^{\circ}$ & $-46^{\circ}$ \\
$-\pi / 4$ & 0.648 & 0.991 & 0.0015 & $15^{\circ}$ & $-38^{\circ}$ & $-35^{\circ}$ \\
0 & 0.629 & 0.996 & 0.0008 & $-4^{\circ}$ & $0^{\circ}$ & $0^{\circ}$ \\
$\pi / 4$ & 0.639 & 0.991 & 0.0012 & $-17^{\circ}$ & $38^{\circ}$ & $35^{\circ}$ \\
$\pi / 3$ & 0.649 & 0.961 & 0.0015 & $-25^{\circ}$ & $49^{\circ}$ & $46^{\circ}$ \\
\hline$\pi / 2$ & 0.672 & 0.80 & 0.0023 & $-46^{\circ}$ & $70^{\circ}$ & $66^{\circ}$ \\
$3 \pi / 4$ & 0.699 & 0.35 & 0.0036 & $-85^{\circ}$ & $104^{\circ}$ & $101^{\circ}$ \\
$\pi$ & 0.726 & 0.08 & 0.0043 & $-178^{\circ}$ & $180^{\circ}$ & $180^{\circ}$ \\
\hline \hline
\end{tabular}

TABLE XIV. Oscillation Parameters for Point $(\mathrm{H})$

\begin{tabular}{|c|c|c|c|c|c|c|}
\hline \multirow[b]{2}{*}{$\phi^{\prime}$} & \multicolumn{2}{|c|}{ Predictions: } & $\times 10^{-5} \mathrm{eV}^{2}$, & $\Delta m_{32}^{2}=$ & $0^{-3} \mathrm{eV}^{2}$ & \multirow[b]{2}{*}{$\chi_{2}$} \\
\hline & $\sin ^{2} 2 \theta_{12}$ & $\sin ^{2} 2 \theta_{23}$ & $\sin ^{2} 2 \theta_{13}$ & $\delta_{C P}$ & $\chi_{1}$ & \\
\hline$-\pi$ & 0.746 & 0.17 & $\overline{0.0090}$ & $181^{\circ}$ & $-180^{\circ}$ & $-180^{\circ}$ \\
\hline$-3 \pi / 4$ & 0.719 & 0.38 & 0.0084 & $101^{\circ}$ & $-113^{\circ}$ & $-111^{\circ}$ \\
\hline$-\pi / 2$ & 0.699 & 0.76 & 0.0068 & $60^{\circ}$ & $-74^{\circ}$ & $-70^{\circ}$ \\
\hline$-\pi / 3$ & 0.678 & 0.919 & 0.0056 & $38^{\circ}$ & $-50^{\circ}$ & $-47^{\circ}$ \\
\hline$-\pi / 4$ & 0.667 & 0.960 & 0.0051 & $28^{\circ}$ & $-39^{\circ}$ & $-36^{\circ}$ \\
\hline 0 & 0.648 & 0.993 & 0.0042 & $-1^{\circ}$ & $0^{\circ}$ & $0^{\circ}$ \\
\hline$\pi / 4$ & 0.659 & 0.960 & 0.0047 & $-29^{\circ}$ & $38^{\circ}$ & $36^{\circ}$ \\
\hline$\pi / 3$ & 0.667 & 0.919 & 0.0051 & $-39^{\circ}$ & $50^{\circ}$ & $47^{\circ}$ \\
\hline$\pi / 2$ & 0.688 & 0.76 & 0.0063 & $-60^{\circ}$ & $74^{\circ}$ & $70^{\circ}$ \\
\hline $3 \pi / 4$ & 0.712 & 0.38 & 0.0080 & $-99^{\circ}$ & $113^{\circ}$ & $111^{\circ}$ \\
\hline$\pi$ & 0.746 & 0.17 & 0.0090 & $-179^{\circ}$ & $180^{\circ}$ & $180^{\circ}$ \\
\hline
\end{tabular}

